Revue internationale P.M.E.

Économie et gestion de la petite et moyenne entreprise

\title{
L'association entre la manifestation de la vision stratégique des propriétaires-dirigeants de PME et la performance de leur entreprise
}

\author{
Jean-Marie Nkongolo-Bakenda, Gérald d'Amboise et Bernard Garnier
}

Volume 7, numéro 1, 1994

URI : https://id.erudit.org/iderudit/1008369ar

DOI : https://doi.org/10.7202/1008369ar

Aller au sommaire du numéro

Éditeur(s)

Presses de l’Université du Québec

ISSN

0776-5436 (imprimé)

1918-9699 (numérique)

Découvrir la revue

Citer cet article

Nkongolo-Bakenda, J.-M., d'Amboise, G. \& Garnier, B. (1994). L'association entre la manifestation de la vision stratégique des propriétaires-dirigeants de PME et la performance de leur entreprise. Revue internationale P.M.E., 7(1), 35-61. https://doi.org/10.7202/1008369ar
Résumé de l'article

L'image mentale que se fait le propriétaire-dirigeant du futur de son entreprise marquerait profondément ïorientation de celle-ci. Plusieurs auteurs ont prôné la nécessité d’une vision stratégique adéquate de la part des dirigeants. Peu d'études empiriques ont toutefois tenté d'examiner l'influence qu'elle peut avoir sur la performance. Une étude auprès de 58 propriétaires-dirigeants de PME québécoises a permis d'approfondir davantage cette question. Les résultats montrent que la nature de l'association diffère quelque peu des attentes. 


\title{
L'association entre la manifestation de la vision stratégique des propriétaires-dirigeants de PME et la performance de leur entreprise
}

\author{
Jean-Marie NKONGOLO-BAKENDA* \\ Gérald D'AMBOISE** \\ Bernard GARNIER ${ }^{\star \star \star}$
}

* Jean-Marie Nkongolo-Bakenda est étudiant au doctorat en sciences de l'administration à l'Université Laval. Il détient une licence en gestion du personnel $\left(2^{\mathrm{e}}\right.$ cycle à l'Université Nationale du Zaïre) et une maîtrise en administration des affaires (Université Laval). Il a enseigné (1978-1990) à l'Université Nationale du Zaïre et s'intéresse actuellement à la gestion stratégique des entreprises, à l'entrepreneurship, aux PME et à la gestion des organisations dans les pays en voie de développement. Adresse: Département de management, Faculté des sciences de l'administration, Université Laval, Québec (Québec) G1K 7P4.

** Gérald d'Amboise, Ph. D., est professeur titulaire rattaché au Département de management de la Faculté des sciences de l'administration de l'Université Laval. Il est comptable agréé et détient un doctorat en administration, spécialisation management de l'Université de la Californie à Los Angeles. Ses divers travaux de recherche et ses principales publications portent sur les problèmes des petites et moyennes entreprises québécoises et leurs pratiques administratives. Adresse: Département de management, Faculté des sciences de l'administration, Université Laval, Québec (Québec) G1K 7P4.

*** Bernard Garnier est professeur titulaire et directeur du Département de management de la Faculté des sciences de l'administration de l'Université Laval. Détenteur d'un $\mathrm{Ph}$. D. de l'Université Western, Ontario, il est membre du Groupe de recherche sur la PME et l'entrepreneurship. Ses travaux de recherche portent sur l'entrepreneurship et la création d'entreprises, la gestion des PME et la formation des dirigeants. Il a publié dans diverses revues nord-américaines et européennes dont Journal of Small Business Management, Journal of Private Enterprise, Journal of Small Business and Entrepreneurship, Frontiers of Entrepreneurship Research, Relations industrielles, Gestion 2000, Gérer et comprendre, Enseignement et gestion. Adresse: Département de management, Faculté des sciences de l'administration, Université Laval, Québec (Québec) G1K 7P4.

Ce texte est principalement basé sur les résultats de l'essai de maîtrise en administration des affaires que le premier auteur a réalisé à partir des données du projet de recherches des deux autres auteurs. Ces derniers avaient agi respectivement comme directeur et premier lecteur de l'essai. Les auteurs tiennent à remercier le Conseil de recherches en sciences humaines du Canada pour le financement du projet. 


\title{
RÉSUMÉ
}

L'image mentale que se fait le propriétaire-dirigeant du futur de son entreprise marquerait profondément l'orientation de celle-ci. Plusieurs auteurs ont prôné la nécessité d'une vision stratégique adéquate de la part des dirigeants. Peu d'études empiriques ont toutefois tenté d'examiner l'influence qu'elle peut avoir sur la performance. Une étude auprès de 58 propriétaires-dirigeants de PME québécoises a permis d'approfondir davantage cette question. Les résultats montrent que la nature de l'association diffère quelque peu des attentes.

\begin{abstract}
According to many authors, envisioning the future helps to achieve results. Nevertheless few studies have shown the relationship between strategic vision and performance in business firms. The present paper reports on such an attempt. The study was conducted in 58 Quebec SMEs. The results show that the association is more complex than expected.
\end{abstract}

\section{RESUMEN}

La imagen mental que haga el empresario del futuro de su empresa marcara profundamente el futuro de la misma. Numerosos autores han predicado la necesidad de una visión estratégica adecuada por parte de los empresarios. Muy pocos estudios práctico han tratado de examinar la influencia de la visión sobre los resultados. Un estudio llevado a cabo con 58 empresarios de PMEs quebequenses permitió profundizar mucho más sobre el tema. Los resultados muestran que la relación no es como se esperaba.

\section{Introduction}

La vision stratégique du dirigeant, c'est-à-dire l'image mentale qu'il se fait de l'état futur et souhaitable de son entreprise, est actuellement considérée par plusieurs auteurs comme l'un des principaux facteurs de succès d'une entreprise. Cette importance attachée à la vision stratégique du dirigeant laisse supposer son association positive à la performance de l'entreprise. Cependant, malgré l'abondante littérature sur le sujet, cette association positive n'est pas suffisamment démontrée par des études empiriques. En effet, même au niveau de la grande entreprise, plusieurs auteurs qui s'intéressent à ce type de relation l'énoncent de façon prescriptive et, donc, normative. D'autres auteurs la déduisent par simple constatation soit de la présence de la vision chez les dirigeants des entreprises ayant réussi, soit de son absence chez ceux dont les entreprises ont échoué. L'association positive «vision stratégique-performance» est donc posée, mais elle n'est pas adéquatement vérifiée.

Pourtant, Conger (1990) fait observer que la simple présence de la vision chez le leader ne conduit pas forcément à la performance. Cet auteur affirme que les qualités habituellement considérées comme distinctives du leader efficace 
mènent même parfois ces derniers à des résultats désastreux. Ainsi, en est-il du leader visionnaire qui commet certaines erreurs, notamment la création d'une vision reflétant ses propres besoins plutôt que ceux du marché, la mauvaise appréciation des ressources nécessaires à la réalisation de sa vision, l'appréciation déficiente ou exagérée des besoins du marché et le manque de discernement des changements de l'environnement pour réajuster la vision.

Cette observation de Conger démontre la nécessité d'approfondir empiriquement la compréhension de l'association entre la vision stratégique du dirigeant et la performance de son entreprise. Le présent article est une contribution à cet effort de compréhension à partir des questions suivantes: Quelle est la nature et la force de l'association entre la vision stratégique du dirigeant et la performance de l'entreprise ? Quel niveau de manifestation de la vision stratégique produit la meilleure performance?

\section{Recherches antérieures}

Depuis quelques années, plusieurs chercheurs s'intéressent au concept de vision stratégique. Cet intérêt pour le concept de vision n'est pas sans liens avec ce que certains ont appelé «le miroir du management japonais » (Pascale et Athos, 1984). En effet, à la suite du succès des entreprises japonaises, plusieurs chercheurs et praticiens du management préconisent l'adoption, par les entreprises du monde occidental, de certaines pratiques de direction des entreprises japonaises. Parmi ces pratiques se trouve la prévision à long terme (Keys et Miller, 1984). Ainsi Drucker (1981) attribuait le succès du management japonais à l'accent mis sur le long terme. De même, un dirigeant japonais, invité à donner des conseils aux dirigeants nord-américains, a soutenu que «[...] Je pense que le management américain devrait s'appuyer sur des perspectives de long terme plutôt que s'en tenir uniquement à des objectifs de profit à court terme [...]» (Fortune, 1981). Parlant de ces visions à long terme dans les entreprises japonaises, Yang (1986: 89) précise qu'elles «marquent plus une direction générale que la volonté d'une stratégie formelle».

Bien que plusieurs auteurs considèrent la vision stratégique comme prédictrice du succès de l'entreprise, la littérature, à notre connaissance, recèle très peu d'études qui lient explicitement ces deux variables. En effet, bon nombre d'auteurs qui s'intéressent à ce type de relation la déduisent par simple constatation de la présence de la vision dans les entreprises ayant réussi. Les études de Peters et Waterman (1983), Bennis et Nanus (1985), Peters (1988), Morgan (1988) et Wilkins (1989), pour ne citer que celles-là, font partie de ce courant. Rockey (1986) fait même état des écrits liant la visualisation avec la thérapie, l'athlétisme, l'art, l'éducation, la créativité ou l'auto-amélioration. 
En fait, peu d'auteurs se sont risqués à émettre clairement l'hypothèse théorique d'un lien entre vision et performance. Néanmoins, plusieurs d'entre eux associent simplement et de façon implicite la vision à la réussite de l'organisation. Conger et Kanungo (1988), par exemple, citent le cas de compagnies ayant éprouvé des échecs stratégiques par absence de vision des leaders en autorité. Pour leur part, Richards et Engel (1986) et Gluck (1984) effectuent le cheminement inverse en expliquant, à l'aide d'exemples, les situations où la vision du dirigeant, transformée en vision commune, a bénéficié à l'entreprise.

Signalons que les études de certains auteurs s'appuient cependant sur une démarche plus rigoureuse pour la démonstration d'une influence possible; c'est le cas de Grinyer, Mayes et Mckiernan (1988), de Moss Kanter (1983) et de Hambrick et Crozier (1985). Ces derniers auteurs, par exemple, analysant les problèmes de gestion des firmes à croissance rapide, ont identifié comme facteurs de réussite, la capacité du dirigeant de voir, d'anticiper les changements et de renforcer constamment la vision stratégique au cœur de l'entreprise.

La recherche de mêmes liens dans le contexte des petites et moyennes entreprises est plus hétéroclite. Elle débute parfois par la construction d'un modèle théorique tel celui de Bird (1988). Cet auteur présente, dans un cadre axé sur la création d'une entreprise, une série d'hypothèses sur les entrepreneurs promis au succès: les entrepreneurs visionnaires. Le lien vision stratégiqueperformance est posé, mais il n'est pas démontré.

Par ailleurs, une certaine démarche inductive est aussi présente chez quelques auteurs qui retracent les effets possibles de la vision par l'observation de la réalité organisationnelle. Filion (1990), par exemple, utilisant des cas d'entreprises, construit un modèle qu'il teste de nouveau à l'aide d'entrevues. Pour cet auteur, l'entrepreneur qui réussit semble être celui qui est capable d'exprimer une vision de ce qu'il désire réaliser. D'une vision émergente, concernée par des idées préliminaires sur les produits ou les services, s'opère la transformation en une vision centrale, où un choix stratégique est effectué.

De leur côté, Gibb et Scott (1985) constatent que l'absence de conscience stratégique chez le dirigeant d'une petite et moyenne entreprise risque de conduire cette dernière vers un chemin sans issue. Quant à Haahti (1989), il s'inspire du modèle de White et Hamermesh ainsi que de celui du groupe de recherche Stratos et propose un modèle qui indique l'influence du contexte et de l'orientation stratégique sur la performance d'une petite et moyenne entreprise. Mais il a limité son étude à la seule analyse descriptive de l'orientation stratégique et n'a pas vérifié tout son modèle.

Carrière (1989), pour sa part, affirme que «Vision et action apparaissent comme complémentaires dans l'explication du comportement stratégique du décideur et, notamment, sur le degré de prédiction possible quant à ses actions 
futures ». Cet auteur pousse plus loin ses travaux et établit une association entre la performance et la vision stratégique. Ses résultats lui permettent de conclure (1990) que la vision stratégique est fortement influencée par les succès et les échecs antérieurs. On peut également mentionner à ce niveau les résultats de quelques travaux sur la planification stratégique, mais qui semblent toutefois avoir de fortes implications au plan de la vision stratégique dans les PME. Il s'agit des travaux de Thurston (1983), d'une part, et de ceux d'Acklesberg et Arlow (1985), d'autre part.

En effet, Thurston (1983) constate, à la lumière de nombreux exemples du monde des affaires, que certains dirigeants de PME ont des plans formels, tandis que d'autres n'ont que des plans qui ne sont pas formulés explicitement et qui n'existent que sous forme d'idées dans l'esprit du patron. Pour cet auteur, la présence ou l'absence de plan formel dans une entreprise n'est pas le principal facteur d'une bonne performance; ce qui compte, c'est la maîtrise de plusieurs variables. Dans ce même ordre d'idées, Acklesberg et Arlow ont constaté que la formalisation du plan n'affecte pas la performance d'une PME: «Nous croyons qu'une planification formalisée peut être de peu d'utilité pour les petites entreprises et peut même entraver leur fonctionnement» (p. 63).

De ce tour d'horizon, il ressort très peu de démonstration claire, au sens théorique du terme, des liens positifs entre la vision stratégique et la performance d'une entreprise. Les auteurs procèdent souvent par un raisonnement a contrario, ou se limitent à expliquer les exemples de réussite, rendant ainsi sous-jacente l'idée de performance. Il y a donc rareté des études empiriques basées sur des données plus fines et établissant de façon claire une liaison entre la manifestation de la vision stratégique et la performance. Retenons toutefois qu'à la lumière des résultats de l'étude de Carrière et des constatations faites par d'autres auteurs, on peut émettre l'hypothèse d'un déterminisme réciproque dans la relation «vision-performance». Cela nous incite à préciser notre cadre conceptuel pour circonscrire notre étude.

\section{Hypothèse de recherche, définition des variables et méthodologie}

\subsection{Hypothèse de recherche}

Au regard des éléments de l'analyse de la littérature mentionnés ci-dessus, on peut émettre l'hypothèse de recherche suivante: il existe une association significative et positive entre la manifestation de la vision stratégique du propriétairedirigeant d'une PME et la performance de son entreprise. Autrement dit, les PME dont les propriétaires-dirigeants ont un niveau élevé de manifestation 
de la vision stratégique réalisent de meilleures performances que celles dont le niveau de manifestation de la vision stratégique des propriétaires-dirigeants est faible.

\subsection{Définition des variables}

Cette hypothèse suggère la présence d'une variable indépendante, la manifestation de la vision stratégique, et d'une variable dépendante, la performance.

\subsubsection{Manifestation de la vision stratégique}

La procédure de détermination de la vision stratégique, aussi bien dans le projet dont relève ce travail que dans les travaux d'autres auteurs, a déjà été largement discutée ailleurs (d'Amboise et Nkongolo, 1993). Il convient toutefois de rappeler que dans leurs travaux, certains auteurs mentionnent les qualités d'une bonne vision stratégique. Parmi eux, on peut retenir, à titre d'exemple, les travaux de Morris, Sashkin, Robbins et Duncan, ainsi que les travaux de Livian et al. Énumérant les exigences indispensables pour que la vision du leader à succès soit ancrée dans l'organisation, Morris (1987) cite le contact personnel, la consistance et la clarté. Pour cet auteur, ces exigences signifient successivement que le leader donne lui-même l'exemple et partage son enthousiasme et son engagement avec les autres à travers l'organisation, qu'il recherche et profite de toutes les occasions pour transmettre le message à ses employés et qu'il crée les symboles, devises et rituels qui transmettent effectivement sa vision stratégique à tous les membres de l'entreprise dans leur travail quotidien. Pour sa part, Sashkin (1988) signale que les leaders expriment et expliquent leurs visions stratégiques à travers les mots et les actions sur trois plans: stratégique, tactique et personnel. Sur le plan stratégique, les leaders développent une formulation claire et concise de leur philosophie organisationnelle. Sur le plan tactique, la vision doit être accompagnée par des actions. Cela signifie que les politiques développées et les programmes mis sur pied dọivent la refléter, les cadres intermédiaires et subalternes doivent être impliqués et, enfin, des ressources tant financières qu'humaines considérables doivent être engagées. Sur le plan personnel, le comportement du leader doit être de nature à pousser les autres membres à adhérer à sa vision. Sashkin (1986) a, par ailleurs, soutenu que la création de la vision implique quatre actions du leader: son expression, son explication aux autres, l'extension de la vision à l'organisation tout entière et, enfin, son utilisation en diverses circonstances. De leur côté, Robbins et Duncan (1988) donnent, entre autres, trois types d'attributs bipolaires de la vision stratégique, à savoir, claire-ambiguë, simple-complexe, consensus-absence de consensus. Livian et al. (1989), quant à eux, énumèrent cinq critères qui peuvent 
être pris en compte dans l'expression de la vision par le créateur du projet. Il s'agit de la force de la vision, sa précision, sa cohérence, son réalisme et la place qu'y occupe le créateur.

Tenant compte des qualités qui reviennent dans les travaux de ces différents auteurs, la manifestation de la vision stratégique du propriétaire-dirigeant est évaluée, dans cette étude, par l'expression qui en est faite, par sa diffusion et par sa concrétisation. L'expression est mesurée selon la clarté et la précision de la vision que le propriétaire-dirigeant exprime, la diffusion selon les critères de l'étendue et du formalisme et, enfin, la concrétisation est mesurée selon l'engagement du dirigeant et son aptitude à estimer la disponibilité des ressources nécessaires. Comme on peut le constater, ces aspects ou moments de la manifestation de la vision sont de nature essentiellement qualitative. Aussi, a-t-il été indispensable de les convertir sous une forme numérique, en vue de les rendre opératoires et, donc, associables à la performance. Cette conversion a d'abord été faite par moment, puis de façon globale.

La mesure de la vision stratégique par moment de sa manifestation est faite en attribuant l'une des valeurs 2,1 ou 0 à chacun des moments où la vision est exprimée par le propriétaire-dirigeant. Ces valeurs correspondent respectivement à une forte, une faible ou une absence de la manifestation de la vision stratégique au moment concerné compte tenu des critères préétablis.

Les résultats de la mesure aux trois moments sont, par la suite, additionnés pour trouver la valeur globale de la manifestation de la vision stratégique. Pour chaque propriétaire-dirigeant, nous avons obtenu une valeur globale représentant le niveau de manifestation de sa vision stratégique. Cette valeur globale peut être placée sur une échelle ordinale de sept degrés ${ }^{1}$.

\subsubsection{Performance}

Les auteurs utilisent indistinctement divers indicateurs pour mesurer la performance des entreprises (Venkatraman et Ramanujan, 1986; Robinson Jr, 1983). Pourtant, les résultats d'une étude menée par Robinson Jr et al. (1984) ont permis de constater qu'à un stade d'évolution donné, le sens et l'intensité de la relation entre la stratégie et la performance peuvent changer, également pour les mêmes entreprises, selon le type d'indicateur utilisé.

Par ailleurs, on sait qu'à un momẹnt donné, tous les secteurs économiques d'un pays n'affichent pas le même rythme de croissance. Par conséquent, la comparaison des valeurs brutes des indicateurs de performance des entreprises

1. Le lecteur intéressé peut obtenir plus de détails sur la procédure auprès des auteurs. 
de deux secteurs différents risque d'être biaisée par les tendances du secteur plutôt que par les réalisations propres de l'entreprise. De plus, la collecte des données sur la performance des entreprises se heurte souvent à la résistance des propriétaires soucieux de garder le secret professionnel de leurs affaires en vue de préserver l'image de leur entreprise ou de prévenir toute concurrence déloyale.

Pour tenir compte de ces trois aspects du problème relatifs aux indicateurs de la performance, cette variable est mesurée dans le présent travail par la variation de la valeur de trois indicateurs de chaque entreprise par rapport aux valeurs correspondantes de leurs secteurs. Il s'agit de la variation relative du nombre d'employés ${ }^{2}$, des ventes ${ }^{3}$ et des profits nets ${ }^{4}$ au cours des récentes années.

\subsection{Méthodologie}

\subsection{1. Échantillon et collecte de données}

L'étude portait sur 60 entreprises manufacturières issues de divers secteurs d'activités et sélectionnées sur une liste de 15000 entreprises manufacturières du Québec. Ces entreprises, situées dans les régions de Québec, de Montréal, de Trois-Rivières et d'Estrie ${ }^{5}$ devaient correspondre à un certain profil. Ainsi, elles n'étaient pas des filiales, étaient dirigées par des personnes qui en étaient partiellement ou totalement propriétaires, n'avaient pas plus de 20 millions de dollars de chiffre d'affaires annuel et vivaient des changements importants de diverses natures, notamment le développement de nouveaux produits, l'augmentation de leur part du marché, l'introduction de nouvelles technologies et l'agrandissement de l'entreprise.

2. La performance selon le nombre d'employés est mesurée par le quotient obtenu en divisant le ratio du changement du nombre d'employés de l'entreprise de 1988 à 1991 par le ratio du changement du nombre d'employés du secteur d'activités de cette entreprise pour la même période.

3. La performance selon les ventes est mesurée par le quotient obtenu en divisant le ratio du changement des ventes de l'entreprise de 1988 à 1990 par le ratio du changement des ventes du secteur d'activités de cette entreprise pour la même période.

4. La performance selon les profits nets est mesurée par la qualité du changement des profits nets de l'entreprise de 1988 à 1990 par rapport au changement des profits nets du secteur d'activités pour la même période.

5 . Signalons qu'en 1987 , les entreprises manufacturières de ces régions représentaient $92 \%$ de l'ensemble des entreprises manufacturières du Québec. 
La collecte de données s'est faite en deux phases.

La première phase a été réalisée principalement en 1988 avec quelques compléments d'information en 1989 par quatre chercheurs (tous professeurs) du Groupe de recherche sur la petite et moyenne entreprise et l'entrepreneurship (GRPME) de l'Université Laval grâce à la technique de l'entretien dirigé. Leurs entretiens avec les dirigeants, d'une durée moyenne d'environ deux heures chacun, ont été enregistrés et retranscrits sur le même type de questionnaire que celui qui a servi de guide à l'entretien.

L'élaboration du guide du questionnaire s'est largement inspirée de la littérature relative au concept de vision stratégique (d'Amboise et Gasse, 1989). Pour retrouver l'idée centrale, stratégique, vers laquelle le propriétaire-dirigeant comptait diriger son entreprise, une quinzaine de questions ouvertes ont été prévues. Elles visaient trois aspects dont le premier se rapportait à la capacité du dirigeant à exprimer ses ambitions pour son entreprise et pour lui-même. Le deuxième aspect abordé par le questionnaire avait trait à la communication des ambitions du dirigeant, ou plutôt de l'image qu'il avait pour le futur de son entreprise, aux autres membres de l'entreprise. Le troisième aspect visé par le questionnaire portait, quant à lui, sur la concrétisation de la vision stratégique. Il s'agissait de la possibilité de réalisation de la vision du dirigeant, des moyens et des ressources que le dirigeant comptait engager ou qui étaient déjà engagés pour la réalisation de sa vision stratégique. Au moment où s'achevait l'analyse des données recueillies lors de la première phase de l'enquête, nous entamions la deuxième phase de collecte des données.

Cette dernière fut réalisée à l'été 1991 simultanément par questionnaire écrit et entretien dirigé de quarante-cinq minutes environ. Les données furent recueillies par trois professeurs (membres de l'équipe de 1988) et quatre étudiants à la maîtrise en administration des affaires et elles portaient sur les informations générales de l'entreprise, le profil décisionnel du propriétairedirigeant, les stratégies réalisées et la performance de l'entreprise. La collecte des données relatives à la performance en 1990/1991 était justifiée par le souci de voir l'impact, ou du moins le début de l'impact, de la vision stratégique exprimée en 1988. Cela est basé sur la supposition que la vision stratégique du dirigeant est susceptible d'influencer, ne fût-ce qu'à moyen terme, les résultats de son entreprise.

Rappelons que le questionnaire sur la performance a permis de déterminer, pour chaque entreprise, les variations respectives du nombre d'employés de 1988 à 1991, des ventes de 1988 à 1990 et des profits nets de 1988 à 1990 . Nous avons préféré observer la variation de ces indicateurs plutôt que leurs valeurs absolues par souci de respecter le secret d'affaires des propriétaires-dirigeants et, ainsi, de réduire leur crainte à livrer l'information. Sur les 60 entreprises, 58 ont fourni les renseignements demandés. 


\subsubsection{Analyse et traitement des données}

L'analyse du questionnaire sur la vision a été faite suivant ses trois dimensions ou moments, à savoir l'expression, la diffusion et la concrétisation. En effet, sur la quinzaine de questions posées, quatre portaient sur la nature de la vision stratégique des propriétaires-dirigeants. Elles permettaient d'amener les dirigeants à exprimer d'abord les ambitions qu'ils avaient pour l'entreprise, puis à donner l'image ou le portrait qu'ils se faisaient de l'entreprise dans les prochaines années. Une lecture attentive des réponses des dirigeants à chaque question a permis d'apprécier le degré de précision et de clarté avec lequel chacun avait exprimé sa vision stratégique. Outre la nature de la vision stratégique, les propriétaires-dirigeants étaient amenés à aborder les aspects de la «diffusion» de leurs visions stratégiques grâce à sept questions et sous-questions contenues dans le questionnaire. L'analyse des réponses à ces questions était réalisée en accordant une attention particulière aussi bien aux personnes associées à la discussion de la vision stratégique qu'aux canaux de diffusion. Enfin, l'analyse des questions en rapport avec la concrétisation de la vision, au nombre de cinq, a été faite de la même façon que dans les cas précédents. Toutefois, on accordait ici davantage d'attention au réalisme de la vision et aux actions amorcées pour sa réalisation. En effet, les questions relatives à cette partie du travail invitaient le dirigeant à donner, entre autres, un exemple concret de l'influence de sa vision stratégique sur les actions de l'entreprise et, aussi, à apprécier son caractère réaliste, ainsi que le temps indispensable pour sa réalisation. Elles permettaient, par ailleurs, de se rendre compte de la capacité du dirigeant à estimer les ressources matérielles et humaines indispensables pour atteindre les objectifs de sa vision stratégique.

La détermination de la manifestation de la vision stratégique était réalisée en deux étapes. Dans la première, la partie du questionnaire sur la vision stratégique était dépouillée de façon globale par deux analystes travaillant séparément. Après quelques lectures des transcriptions des entretiens des dirigeants, chaque analyste a évalué la manifestation de la vision stratégique exprimée par chaque propriétaire-dirigeant. Cette évaluation se faisait en situant la vision stratégique du propriétaire-dirigeant sur une échelle de sept catégories ordinales. Les classements faits séparément par les deux analystes ont été par la suite confrontés pour dégager un classement unique.

Dans la deuxième étape, la détermination du niveau de manifestation de la vision stratégique a été faite de façon analytique par un troisième chercheur, d'abord en indiquant le niveau de la vision à chacun de ses moments, à savoir l'expression, la diffusion et la concrétisation, puis en combinant les scores aux différents moments pour trouver un score global. Ainsi pour toute vision exprimée, chaque moment recevait un score allant de 0 à 2 conformément 
aux critères fixés. La sommation des scores obtenus par la vision stratégique du propriétaire-dirigeant aux trois moments de sa manifestation déterminait son niveau global.

Les niveaux globaux de manifestation des visions stratégiques ainsi obtenus ont été par la suite comparés à ceux trouvés précédemment par les deux analystes. Après avoir confronté ces deux listes de scores globaux, nous avons constaté un écart d'au moins deux points pour les visions stratégiques des propriétaires-dirigeants de 17 entreprises. Les visions stratégiques de ces 17 propriétaires-dirigeants ont été réexaminées de façon analytique soit pour confirmer leurs scores, soit pour les corriger. Nous avons ainsi obtenu pour chaque entreprise la valeur globale du niveau de manifestation de la vision stratégique exprimée par son propriétaire-dirigeant. C'est cette valeur qui a été utilisée pour associer la vision à la performance de l'entreprise.

Le dépouillement des données issues de la deuxième phase de l'étude n'a pas nécessité d'opérations particulières, car les ratios de différents indicateurs étaient directement inscrits par le propriétaire-dirigeant sur le questionnaire qui lui était adressé. De même, les données statistiques relatives aux performances des secteurs d'activités étaient obtenues des Services de Statistique Canada. Elles n'ont pas, elles non plus, nécessité d'opérations particulières de dépouillement.

Le traitement des données a été fait principalement sur ordinateur Macintosh grâce au logiciel Statview 512+. Toutefois, lorsque la formule n'était pas programmée sur ce logiciel, nous avons pu soit créer une macrofonction correspondante grâce au logiciel Excel, soit utiliser le logiciel SPSSX sur ordinateur IBM.

Pour vérifier les hypothèses, nous avons principalement utilisé le graphique et le coefficient de corrélation par rang de Kendall. Le choix de ce coefficient a été justifié, d'une part, par la nature ordinale de la plupart des données et, d'autre part, par la possibilité qu'il offrait de tester la signification des coefficients calculés sur des échantillons de taille même assez réduite (comme $\mathrm{n}<10$ ) (Siegel et Castellan, 1988).

\section{Résultats}

Dans cette section, les résultats du dépouillement des données et ceux des analyses subséquentes sont présentés par indicateur de performance. Ainsi, la manifestation de la vision stratégique du propriétaire-dirigeant est associée successivement au changement relatif du nombre d'employés, au changement relatif des ventes et, enfin, au changement relatif des profits nets. 


\subsection{Association entre le niveau de manifestation de la vision stratégique du propriétaire-dirigeant et le changement relatif du nombre d'employés}

Rappelons que la manifestation de la vision stratégique est mesurée sur une échelle à sept niveaux. Ces derniers s'étendent de l'absence d'expression d'une vision stratégique pour l'entreprise chez le propriétaire-dirigeant à une expression d'une vision diffusée à l'ensemble des employés et ayant même fait l'objet d'une amorce de réalisation. De tous les 60 propriétaires-dirigeants interviewés en 1988, plus de $60 \%$ se sont classés aux trois niveaux supérieurs alors qu'un peu moins de $20 \%$ seulement se sont classés aux trois niveaux inférieurs.

Lors des enquêtes de 1991 sur les mêmes entreprises, on a obtenu les renseignements relatifs au nombre d'employés pour 58 entreprises. À l'issue du dépouillement de ces renseignements, nous avons constaté que 6 entreprises (soit $10 \%$ de l'ensemble) étaient fermées pour cause de faillite ou pour un autre motif, 24 entreprises (soit $42 \%$ ) avaient maintenu ou augmenté le nombre de leurs employés et 28 entreprises (soit $48 \%$ ) avaient connu une diminution du nombre de leurs employés. Au cours de la même période, les secteurs de 5 entreprises seulement (soit $9 \%$ de l'ensemble) avaient maintenu ou augmenté le nombre de leurs employés et les secteurs de 53 entreprises (soit $91 \%$ de l'ensemble) avaient connu une diminution du nombre d'employés. De la comparaison entre la variation du nombre d'employés par entreprise et la variation du nombre d'employés dans le secteur correspondant, il ressort qu'à part les 6 entreprises (soit 10,3\%) fermées, 37 entreprises (soit 63,8\%) ont connu une variation du nombre d'employés égale ou supérieure à celle de leurs secteurs et 15 entreprises (soit $25,9 \%$ ) ont connu une variation du nombre d'employés inférieure à celle de leurs secteurs respectifs ${ }^{6}$.

En déterminant les ratios relatifs de variation du nombre d'employés par niveau de manifestation de la vision stratégique du propriétaire-dirigeant, nous avons obtenu le graphique 1 .

De ce graphique, il ressort que la situation des entreprises ayant réalisé une meilleure performance au regard du nombre d'employés (celles dont la variation du nombre d'employés est égale ou supérieure à celle du secteur) n'affiche pas une tendance linéaire et croissante au fur et à mesure que le niveau de manifestation de la vision stratégique s'élève. D'ailleurs, le tau de Kendall trouvé

6. Sur le graphique, les entreprises fermées se situent à l'échelle 0 et celles qui ont le même indice de variation que leur secteur se situent à l'échelle 100. 
entre les deux variables est de $-0,031$ et correspond au $z$ de $-0,348(p=0,36)$. Il n'est pas significatif au seuil de $10 \%$. Autrement dit, il n'y a pas d'association significative entre la manifestation de la vision stratégique et le changement relatif du nombre d'employés dans les PME étudiées.

\section{GRAPHIQUE 1}

Variation relative du nombre d'employés des entreprises par niveau de manifestation de la vision stratégique du propriétaire-dirigeant

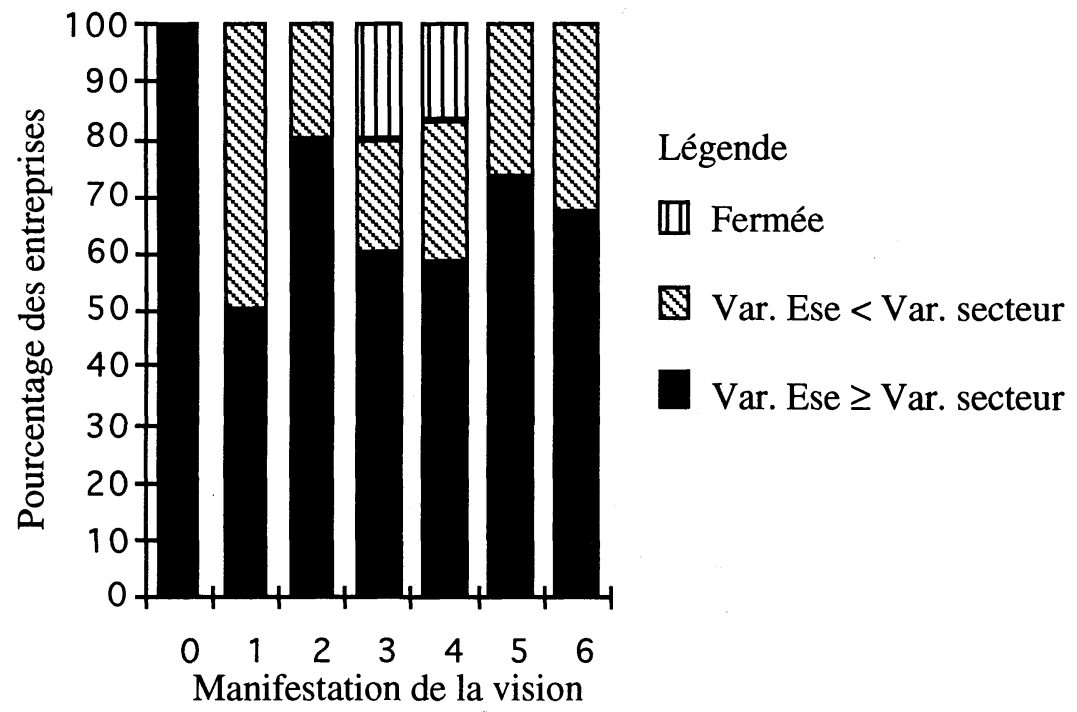

\subsection{Association entre le niveau de manifestation de la vision stratégique du propriétaire-dirigeant et le changement relatif des ventes}

Sur les 58 entreprises pour lesquelles nous avons eu des renseignements relatifs aux ventes, il y en a 6 qui ont fermé (soit 10,3\%) en 1990. La valeur des ventes de 31 entreprises (soit 53,4\%) était, à cette période, égale ou supérieure à celle de 1988 et dans 21 entreprises (soit 36,3\%), la valeur des ventes en 1990 était inférieure à celle de 1988. À la même période, la valeur des ventes des secteurs d'activités n'avait pas atteint son niveau de 1988 dans le cas de 9 entreprises seulement (soit 15,5\% des entreprises étudiées).

De la comparaison entre la variation de la valeur des ventes dans les entreprises et celle de leurs secteurs respectifs, il ressort qu'à part les 6 entreprises fermées, il y en a 24 (soit $41,4 \%$ de l'ensemble) dont la variation des 
ventes de 1988 à 1990 est égale ou supérieure à celle du secteur d'activités et 28 entreprises (soit 48,3\% de l'ensemble) dont la variation des ventes, pour la même période, est inférieure à celle des secteurs respectifs d'activités.

En déterminant les ratios relatifs de variation de ventes par niveau de manifestation de la vision stratégique du propriétaire-dirigeant, nous avons obtenu le graphique suivant.

\section{GRAPHIQUE 2}

Variation relative des ventes des entreprises par niveau de manifestation de la vision stratégique du propriétaire-dirigeant

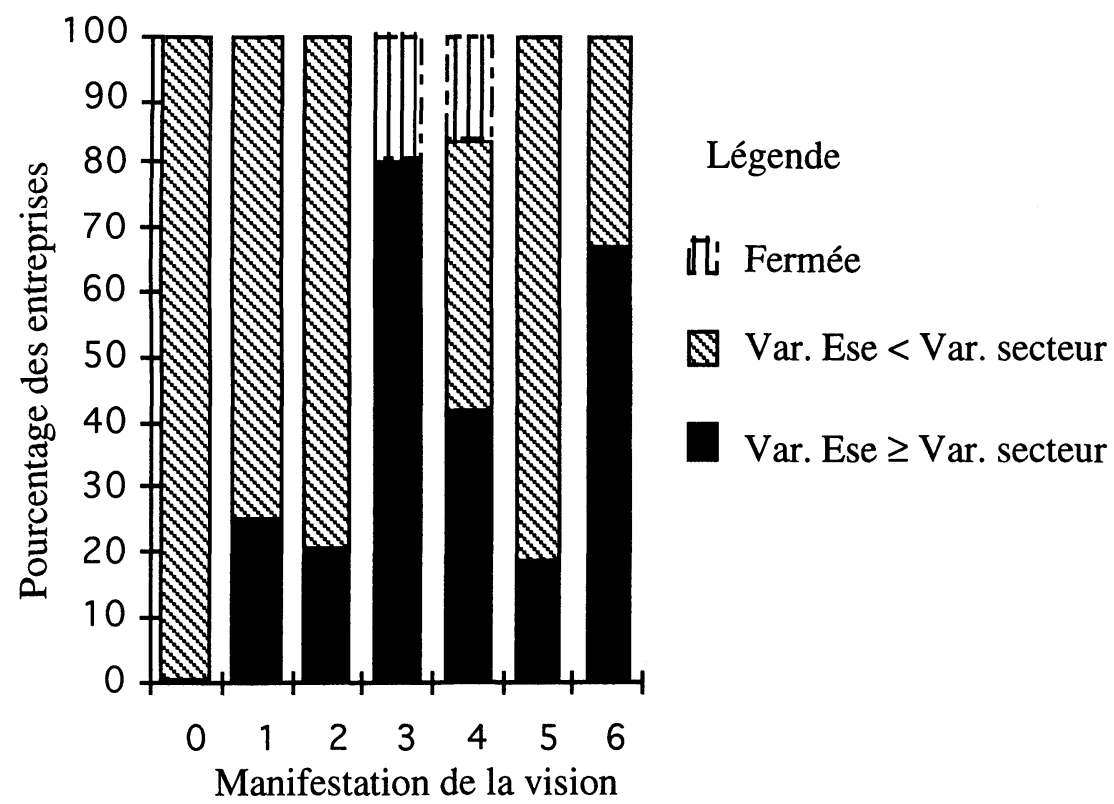

Sur ce graphique, on ne constate pas une tendance positive et croissante entre le nombre d'entreprises performantes (celles dont la variation des ventes est égale ou supérieure à celle du secteur) et le niveau de manifestation de la vision stratégique comme le suggérait notre hypothèse de recherche. Le tau de Kendall entre la manifestation de la vision stratégique et le ratio relatif des ventes est de $-0,041$ correspondant au $z$ de $-0,45(p=0,33)$ qui n'est même pas significatif au seuil de décision de $10 \%$. On peut donc affirmer qu'il n'y a pas d'association significative entre le niveau de manifestation de la vision stratégique du propriétaire-dirigeant et la variation relative des ventes de l'entreprise. 


\subsection{Association entre le niveau de manifestation de la vision stratégique du propriétaire-dirigeant et le changement relatif des profits nets}

Lors des enquêtes de 1991, nous avons obtenu les renseignements relatifs aux profits nets pour 58 entreprises. De ces dernières, 17 (soit 29,3\%) avaient connu une perte. Il convient de signaler que 3 de ces 17 entreprises furent fermées pour cause de faillite. Par ailleurs, 5 entreprises (soit 8,6\%) avaient, en 1990, un résultat nul soit parce qu'elles étaient fermées sans qu'il y ait faillite (cas de trois entreprises), soit parce que tel était leur résultat réel (cas de 2 entreprises). À part les cas précédents, il y avait 20 entreprises (soit 34,5\%) dont les profits nets en 1990 n'avaient pas atteint leur valeur de 1988 et 16 entreprises (soit $27,6 \%$ ) dont la valeur des profits nets en 1990 avait atteint ou dépassé celle de 1988. Signalons en passant que sur les 58 entreprises étudiées, il n'y en avait qu'une seule (soit $1,7 \%$ ) pour laquelle la valeur des profits nets du secteur d'activités avait, en 1990, atteint son niveau de 1988.

De l'examen de la variation des profits nets des entreprises et de leurs secteurs respectifs, il ressort que les 16 entreprises (soit 27,6\%) ayant en 1990 des bénéfices d'une valeur supérieure à celle de 1988 ont également connu une variation des profits nets supérieure à celle de leurs secteurs d'activités. Il s'est également dégagé que 11 entreprises (soit $19 \%$ des entreprises étudiées), malgré une réduction de leurs bénéfices en 1990 par rapport à 1988, ont connu une variation des bénéfices égale ou supérieure à celle de leurs secteurs respectifs. Par ailleurs, il y a aussi 14 entreprises (soit $24,1 \%$ ) qui ont connu une réduction des bénéfices plus importante que celle de leurs secteurs respectifs. Enfin, les 17 autres entreprises restantes (soit 29,3\%) ont vu leur déficit de 1988 s'aggraver encore davantage en 1990 . Nous avons donc quatre catégories d'entreprises: amélioration du bénéfice plus importante que le secteur, régression du bénéfice moins importante que le secteur, régression du bénéfice plus importante que le secteur et, enfin, perte.

En déterminant le type de variation relative des profits par niveau de manifestation de la vision stratégique du propriétaire-dirigeant, nous avons obtenu le graphique 3 .

De ce graphique, si l'on considère la situation des entreprises qui ont une bonne performance relative (performance 3 et 4 , soit les entreprises dont la variation des profits est égale ou supérieure à celle du secteur), on ne constate pas une tendance linéaire et croissante. Cependant, le calcul de la corrélation de rang de Kendall entre la manifestation de la vision stratégique et la variation relative des profits nets a donné un tau de $-0,222$ correspondant au $z=-2,466$ ( $\mathrm{p}=0,014$ ) significatif au niveau $5 \%$ pour un test bilatéral. Il semble utile de faire remarquer que le signe négatif du coefficient est dû au fait que le maximum le 


\section{GRAPHiQue 3}

Variation relative des profits des entreprises par niveau de manifestation de la vision stratégique du propriétaire-dirigeant

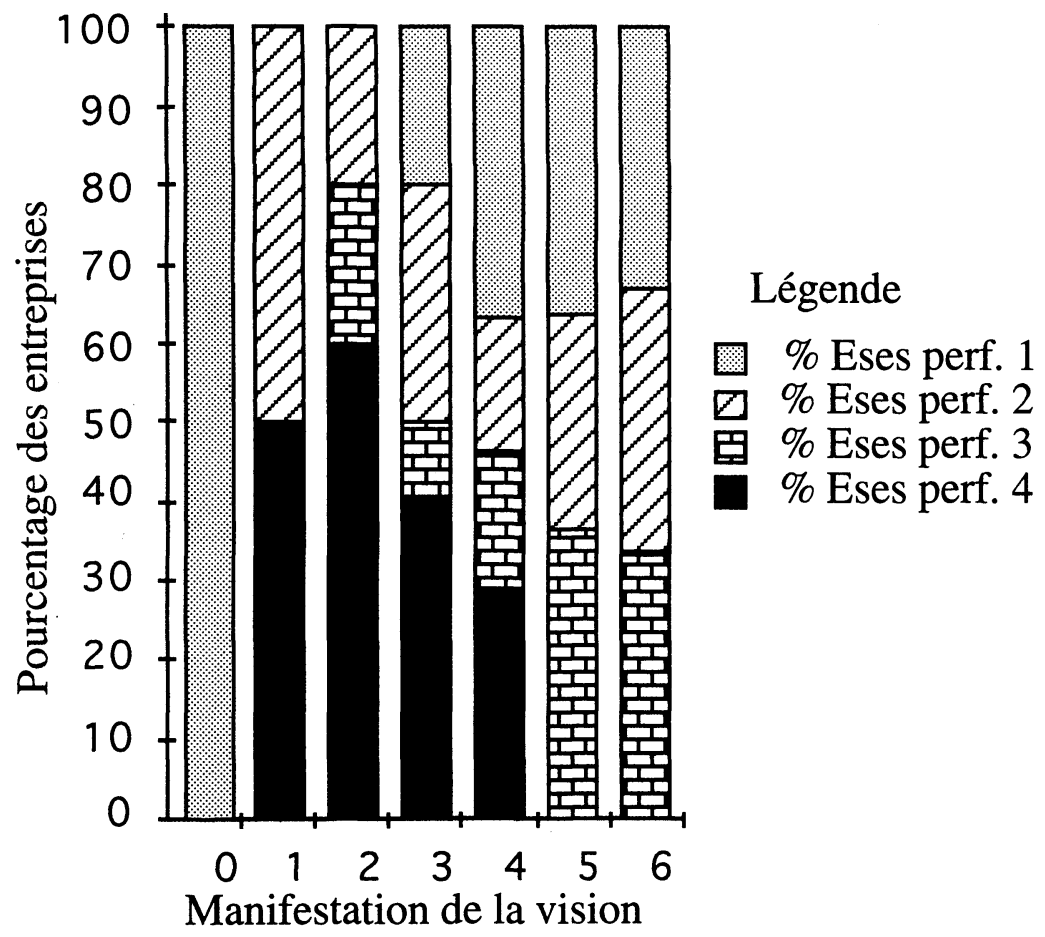

plus élevé de la courbe se situe aux niveaux inférieurs de manifestation de la vision stratégique (niveau 2) et non au niveau 6, qui est le degré ultime de la manifestation de la vision stratégique. De plus, on se souvient que plus de $60 \%$ des entreprises étudiées se situent aux niveaux de vision stratégique 4 et 5. Étant donné que plusieurs entreprises de ces niveaux n'ont pas réalisé une bonne performance, il est normal que la courbe n'y soit pas à des valeurs élevées de performance. Si l'on pouvait se permettre de procéder à l'ajustement des nuages des points de l'association entre la manifestation de la vision stratégique et la variation relative des profits nets, nous aurions le graphique 4 .

Sur ce graphique, l'association entre la manifestation de la vision stratégique et la variation relative des profits est représentée par une courbe de régression de troisième degré $(\mathrm{p}=0,05)$, curvilinéaire, croissante aux niveaux de manifestation de la vision stratégique $0-2$ et 5-6, mais décroissante aux niveaux 2-5. Il est intéressant de constater qu'en dépit de sa tendance croissante au niveau 6 de manifestation de la vision stratégique, la performance n'atteint pas 
la valeur maximale de performance réalisée au niveau 2 de manifestation. Ainsi, il s'avère que la meilleure performance est réalisée aux niveaux 2 de manifestation de la vision stratégique plutôt qu'au niveau 6 . Il convient de souligner le fait que le niveau 2 de manifestation de la vision stratégique correspond à une expression du futur de l'entreprise étayée par des buts ou des objectifs stratégiques explicites parfois décomposés en étapes. À ce niveau, même la procédure de réalisation du rêve du dirigeant est quelquefois envisagée malgré l'absence du souci de diffusion. Quant au niveau 6 de manifestation de la vision stratégique, il correspond à une vision qui, non seulement est clairement exprimée et fortement diffusée à l'ensemble des employés, mais fait en plus l'objet d'une concrétisation allant même jusqu'à la réalisation de certaines de ses phases.

\section{GRAPHIQUE 4}

Nuage de points et courbe de régression entre le niveau global de vision stratégique et la variation relative des profits nets ${ }^{7}$

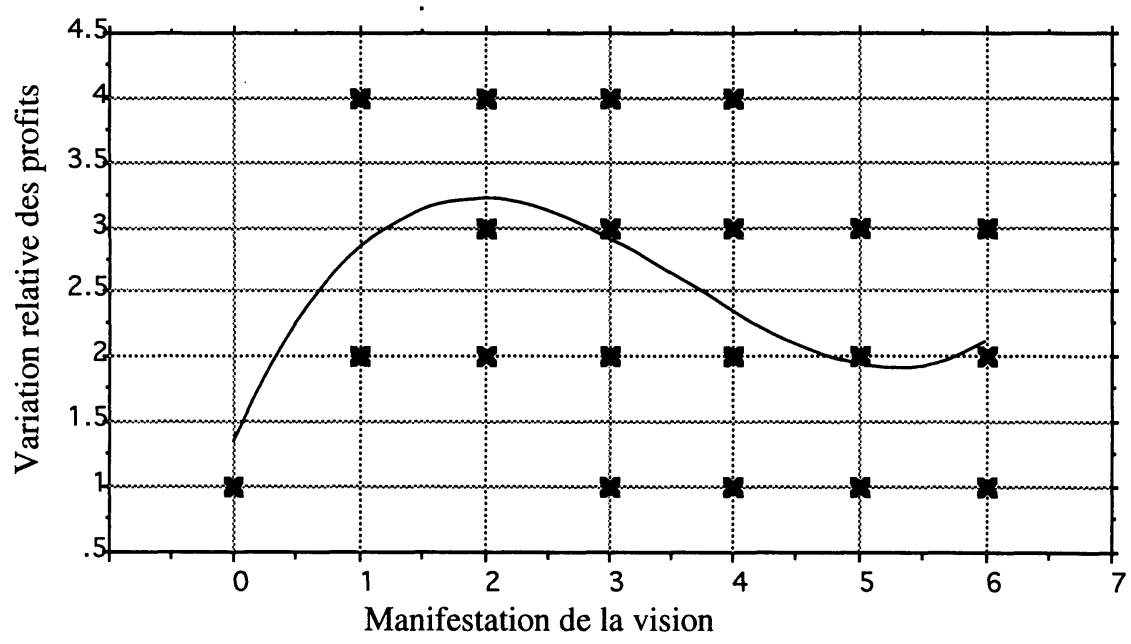

La courbe d'association du graphique 4 montre, sous une forme continue, la situation constatée au graphique 3. Elle est non monotone et ne s'accommode donc pas toujours des traitements de type linéaire (Blalock, 1979). C'est pourquoi nous avons également calculé, en plus du coefficient du tau de Kendall

7. Signalons que le niveau descriptif du test de signification pour cette courbe donne la probabilité $\mathrm{p}$ de 0,05 , mais c'est la plus faible car pour le premier, deuxième et quatrième degré, on obtient successivement les $p$ de $0,08,0,11$ et 0,09 . 
ci-dessus, le rapport de corrélation êta entre les deux variables ${ }^{8}$. On pourrait s'objecter à l'utilisation de ce rapport étant donné que la variable dépendante (VRP) est exprimée en rang. Pour répondre à cette objection, rappelons qu'il s'agit ici des rangs des catégories (ou des classes) et non des rangs des données individuelles. Bialès (1988) signale à ce sujet que :

Pour déterminer les rapports de corrélation, les variances conditionnelles sont indispensables. Or, le calcul de celles-ci n'est possible que dans le cas de données regroupées en classes. Lorsqu'un tel regroupement en classes ne peut pas être envisagé, et que l'on raisonne par conséquent sur une population décrite individu par individu [...], seul le coefficient de corrélation linéaire peut être calculé.

Dans le cas présent, les profits nets sont regroupés en quatre classes et chacune de celles-ci, au lieu d'être représentée par le point-milieu, est plutôt représentée par le rang. D'ailleurs, le rapport de corrélation est calculé sur un tableau de contingence ayant deux séries de rangs correspondant, d'un côté, aux niveaux de manifestation de la vision stratégique et, de l'autre, aux classes des profits nets.

Les calculs ont donné un rapport êta de 0,39 qui correspond au t de student de 3,08 (df = 53) et, donc, qui est significatif au seuil de $1 \%$. Nous pouvons affirmer, à la lumière de ce résultat, que l'association entre le niveau de manifestation de la vision stratégique et la variation relative des profits nets est très élevée. Elle est toutefois de nature curvilinéaire.

\section{Discussion}

Les résultats ci-dessus sont modestes par rapport aux attentes de notre hypothèse de travail. Ils sont d'autant plus surprenants que la littérature organisationnelle récente sous-entend l'existence d'une forte association linéaire et positive entre la vision stratégique et la performance. Bennis (1990), par exemple, n'affirmaitil pas que «Actuellement, la vision est une chose fragile qui a besoin d'être nourrie et développée chez la direction, les employés et les cadres [...] Une des plus grandes menaces pour les entreprises américaines est l'étroitesse de leur horizon ou leur tendance à restreindre leur horizon [...]» ? Cependant,

8. Le rapport de corrélation est une généralisation du coefficient de corrélation puisqu'il peut s'appliquer même dans les cas où la régression n'est pas linéaire (Mialaret, 1991). C'est un indice de la valeur de la prédiction d'une variable à partir d'une autre lorsque la relation entre ces variables n'est pas linéaire (D'Hainaut, 1978). 
l'étonnement provoqué par le résultat devrait être atténué, car dans la revue de la littérature, on avait souligné l'insuffisance des recherches empiriques publiées sur l'association «vision stratégique-performance». En plus, on sait que l'existence de cette association était essentiellement soutenue de façon prescriptive et implicite. Aussi, plutôt que d'être décourageants, les résultats trouvés incitentils à plus de réflexion et d'analyse pour mieux connaître le rôle réel de la vision stratégique dans la gestion d'une PME. Dans cette section, un effort a été fait dans ce sens pour commenter les résultats trouvés à la lumière de certains éléments de la littérature. Ces commentaires sont faits séparément pour chaque indicateur de performance.

\subsection{Association entre la manifestation de la vision stratégique et la performance selon le nombre d'employés}

Le caractère non significatif de l'association entre la manifestation de la vision stratégique et la variation relative du nombre d'employés, à première vue surprenant, peut cependant s'expliquer aussi bien par rapport aux faits que par rapport à la théorie.

Sur le plan des faits, plusieurs spécialistes estiment que les PME québécoises accordent une attention particulière à l'équipement technologique (Les Affaires, 19 octobre 1991). Dans un tel contexte, les entreprises accordent beaucoup plus d'importance à la compétence et, donc parfois à la formation pour une meilleure adaptation aux nouvelles machines, qu'à l'accroissement du nombre d'employés. D'ailleurs, l'informatisation croissante des tâches et le souci de réduire les coûts pour être compétitif ne peuvent pas favoriser l'accroissement du nombre d'employés par entreprise, de sorte que la variation du nombre d'employés, bien qu'elle puisse être un indicateur de l'envergure des opérations de l'entreprise et de son impact au niveau de la communauté en général, n'est pas toujours le reflet de l'importance de la performance du point de vue de l'entreprise elle-même.

Sur le plan de la théorie, la mesure du succès de la mise en place d'une stratégie par la variation du nombre d'employés sous-entend un lien entre ces deux variables. Cependant, ce lien, même s'il existe, ne se traduit pas forcément par l'accroissement du nombre d'employés (Miller, 1985). Du reste, l'existence d'un tel lien n'est pas toujours évidente. Ainsi, certains auteurs constatent que «les moyens de RH [ressources humaines ] [...] ne varient pas beaucoup avec le type de stratégie [...], pas plus que l'importance accordée aux diverses activités de GRH [gestion de ressources humaines] par les dirigeants d'entreprises ayant des stratégies différentes » (Wils et al., 1989). Pour ces auteurs, «certains 
systèmes de GRH peuvent ne varier ni en fonction des stratégies d'entreprise, ni en fonction des catégories d'employés, mais reflètent plutôt un autre facteur, à savoir, l'existence d'une politique sociale de l'entreprise» (Wils et al., 1989). Cette conception est proche de celle de Sorge et Maurice (1990) lorsqu'ils affirment, parlant de l'efficacité des employés, que «[...] leur efficacité [...] varie en fonction des tâches ou du contexte industriel où ils se trouvent. De plus, celle-ci relève non seulement de la performance effective dans une situation spécifique mais aussi dans un cadre institutionnel problématique non spécifique».

Compte tenu, d'une part, du caractère innovateur (dans le domaine technologique) des PME québécoises étudiées et, d'autre part, de la nature contingente du lien «stratégie-ressources humaines», on peut comprendre que l'association «manifestation de la vision stratégique-variation du nombre d'employés» n'ait pas été significative dans le cadre de cette étude.

\subsection{Association entre la manifestation de la vision stratégique et la performance selon les ventes}

Le caractère non significatif de l'association «manifestation de la vision stratégique-variation des ventes » peut être expliquée par le contexte économique du Québec pendant la période de l'étude. En effet, cette période a coïncidé avec la récession qui a commencé en 1989 (MICT, 1991). Dans un tel contexte, les entreprises accroissent le contrôle de leurs dépenses et n'entreprennent que les projets pour lesquels ils ont plus d'assurance de succès. Elles veillent donc davantage à la survie qu'à l'extension des activités. Ainsi, les meilleures entreprises sont celles qui vendent en visant le plus de profits possible.

C'est, à notre avis, cette situation qui s'est produite dans le cas qui nous intéresse. En effet, on se souviendra que les entreprises étudiées ont été choisies parmi les plus dynamiques de différents secteurs. Or, nous avons constaté qu'alors que $36 \%$ des entreprises étudiées avaient connu une diminution de leurs ventes pendant la période d'étude, il y avait seulement $15,5 \%$ des entreprises dont les ventes de secteurs d'activités avaient diminué. Par ailleurs, alors que $29,1 \%$ des entreprises étudiées avaient connu une augmentation de leurs bénéfices, il n'y avait que $1,7 \%$ des entreprises dont le secteur d'activités avait connu une augmentation de bénéfices au cours de la même période. Cela montre donc qu'en dépit de la diminution de leurs ventes, les entreprises étudiées ont, dans l'ensemble, réalisé des bénéfices dans des proportions supérieures à celles de leurs secteurs respectifs.

L'utilisation des ventes de l'entreprise comme indicateur de performance est ambiguë. Les variations des ventes sont, en effet, contextuelles à la situation propre de chaque entreprise. Ainsi, pour certaines entreprises, l'augmentation des ventes reflète le succès alors que pour d'autres, l'absence d'augmentation 
des ventes ne traduit pas forcément une mauvaise performance. Par conséquent, en réunissant des entreprises issues de réalités contextuelles différentes comme dans le cas présent, l'association «manifestation de la vision stratégiqueperformance» peut ne pas être significative.

\subsection{Association entre la manifestation de la vision stratégique et la performance selon les profits nets}

Bien que la littérature organisationnelle suggérât l'existence d'une association positive et linéaire entre la manifestation de la vision stratégique et les profits nets, les résultats de cette étude ont montré qu'il existe bien une certaine association entre les deux variables, mais que celle-ci, plutôt que d'être linéaire, est curvilinéaire de type non monotone. Par ailleurs, nous avons également constaté que la seule présence de la vision à un niveau donné n'est pas suffisante en soi pour assurer la meilleure performance. Pour cela, il faudrait que le niveau de manifestation de la vision stratégique soit en adéquation avec les autres facteurs organisationnels.

Ce constat des limites du pouvoir prédicteur de la performance par la vision stratégique rejoint certaines réserves exprimées par Conger (1990). Toutefois, il faut remarquer qu'alors que cet auteur situait ces limites au niveau du contenu de la vision stratégique, la présente étude donne un nouvel éclairage sur les limites de l'intensité de la vision stratégique à pouvoir prédire la performance de l'entreprise. Pour mieux comprendre la portée de ces résultats, il semble utile de rappeler le constat fait par certaines études sur l'association «planification stratégique-performance».

On se rappellera que la vision stratégique était souvent présentée comme une alternative à la rigidité du processus «rationnel» de planification stratégique en vue de faire face à la turbulence environnementale ainsi qu'à l'insuffisance des moyens matériels et financiers dans les PME. Pour Ackelsberg et Arlow (1985), «Trop de formalisme peut limiter la flexibilité et le mouvement entrepreneurial des [...] firmes». C'est également dans ce sens qu'abonde Thurston (1983) lorsqu'il affirme, en parlant de la relation «planification stratégiqueperformance» que «La présence ou l'absence de planification formelle ne semble pas être une variable majeure [...]. En fait, aucune variable n'est déterminante. Toutes interagissent ». En ce qui concerne la vision stratégique telle qu'elle a été mesurée dans ce travail à la lumière de la littérature qui la concerne, on peut dire qu'elle n'a pas elle-même échappé au danger de rigidité.

En effet, les résultats de ce travail ont révélé une meilleure performance pour les entreprises dont le niveau de manifestation de la vision stratégique était de 2. Il s'agit d'entreprises dont le dirigeant a exprimé une vision stratégique claire et précise, mais sans se préoccuper de sa diffusion ou de sa concrétisation. 
Par contre, les entreprises dont les visions des propriétaires-dirigeants se situaient aux niveaux «5-6» n'ont pas réalisé généralement une très bonne performance. Ces niveaux correspondent à des visions stratégiques qui, non seulement sont exprimées de façon claire et précise, mais en plus sont largement diffusées, formalisées et, même, concrétisées. Compte tenu de la récession qui a suivi l'année au cours de laquelle ces visions ont été exprimées (1988), il y a lieu de se demander si ce n'est pas l'excès de formalisme qui aurait empêché leur adaptation aux nouvelles conditions environnementales. Une récession entraîne, en effet, une certaine incertitude. C'est pourquoi les niveaux supérieurs de vision stratégique ne semblent pas appropriés au contexte, car ils supposent une démarche irréversible alors que les niveaux 1-3 permettent une certaine flexibilité par rapport à l'environnement.

\section{Limites de l'étude et pistes de recherche}

Les résultats de cette étude ont certaines limites.

D'abord, il faut dire que l'étude porte sur certaines des PME manufacturières parmi les plus dynamiques du Québec, et qu'il serait hasardeux d'en généraliser les résultats. Deuxièmement, cette étude a été faite pendant une période de récession. En effet, les données sur la vision stratégique ont été recueillies en 1988, année au cours de laquelle la situation économique était florissante. Or, comme le note le MICT (1991) dans l'une de ses publications, «après le sommet atteint en 1988, les profits des entreprises de fabrication ont subi des baisses successives de $8,9 \%$ en $1989,30,8 \%$ en 1990 et $56,8 \%$ en 1991 ». Dans cette situation, il n'est pas évident que les visions exprimées en 1988 soient demeurées inchangées par la suite. De plus, les entreprises choisies étaient dans des phases d'importants changements et il est fort possible qu'au bout de deux ans, ceux-ci n'aient pas encore apporté de résultats escomptés. Nous pointons là le problème, auquel la littérature organisationnelle n'a pas encore trouvé de solution, de la durée de la période nécessaire pour mesurer la performance (Hrebiniak, Joyce et Snow, 1989).

En troisième lieu, il faut remarquer que la nature même des variables étudiées constitue en soi une limite aux résultats de cette étude. En effet, la vision stratégique du propriétaire-dirigeant est une variable peu tangible. Sa saisie plus complète aurait peut-être nécessité une longue présence du chercheur dans l'entreprise. Par ailleurs, la performance de l'entreprise est mesurée selon les mêmes indicateurs pour toutes les entreprises. Pourtant, il n'est pas évident que ces dernières aient eu les mêmes objectifs, ou du moins les mêmes préoccupations, au moment de l'étude. De plus, la mesure de cette performance s'est limitée à quelques indicateurs. Or, comme le signalent Hrebiniak et al. (1989), la performance est un résultat multidimensionnel de l'évaluation du comporte- 
ment organisationnel. On peut donc, avec raison, appliquer au secteur organisationnel cette affirmation avancée par Nadeau (1988) dans le secteur de l'éducation, à savoir que «la simple assignation de nombres à une performance est un acte neutre qui ne dit pas si celle-ci est bonne ou mauvaise ». Peut-être que les entreprises considérées, selon les indicateurs, comme ayant une mauvaise performance ne le sont qu'en apparence pour un horizon limité. Du reste, les facteurs à la base de la performance des organisations sont tellement nombreux et imbriqués les uns dans les autres que l'isolement de l'influence d'une seule cause est une entreprise difficile.

Compte tenu des limites qui viennent d'être énumérées, il s'avère utile que la présente étude soit répétée sur une période plus longue tant sur les mêmes entreprises que sur les entreprises d'autres milieux. Dans ce cadre, l'approche par les études de cas semble appropriée pour une meilleure compréhension des différentes variables en présence. Il s'avère également utile qu'à l'avenir, les chercheurs intéressés par la vision stratégique se penchent sur les facteurs qui favorisent la performance des entreprises à chaque niveau de manifestation de la vision stratégique. Il s'agit donc d'approfondir la connaissance du rôle de la vision stratégique dans la congruence de diverses variables organisationnelles. Nous pensons que l'ensemble du projet dont fait partie la présente étude apportera déjà une certaine contribution dans ce sens.

\section{Conclusion}

La vision stratégique du propriétaire-dirigeant est un concept qui suscite un intérêt croissant et la portée exacte de ses effets sur l'organisation mérite un examen empirique et approfondi. Cette étude a montré qu'elle (la vision stratégique) est porteuse d'espoir pour un certain succès de l'entreprise. Cependant, elle n'est pas une panacée, son intensité elle-même n'est pas sans restrictions pour des résultats prometteurs. Cela suppose qu'une certaine vision est nécessaire pour une meilleure performance, mais qu'une très forte vision ne facilite pas toujours l'adaptation, car toute vision renferme quelque chose d'illusoire. Peut-être que son analyse est plus exigeante que ce qui a pu être réalisé ici : il s'agit d'un premier essai à la recherche d'une association. Il est également fort probable que les effets d'une vision stratégique exprimée, diffusée et concrètement amorcée excèdent les indicateurs financiers traditionnels de résultats d'une entreprise observés sur une courte période.

Ainsi, la problématique est complexe. Les divers aspects de la présente étude auront certainement aidé à mieux comprendre le concept de vision stratégique; mieux en constater les effets demeure toutefois un défi majeur. Que cette tentative d'identifier l'existence et l'influence de la vision stratégique de quelques propriétaires-dirigeants de PME puisse inspirer de nouvelles initiatives! 


\section{Bibliographie}

ACKELSBERG, R. et P. ARLOw (1985), «Small business do plan and it pays off », Long Range Planning, vol. $18, \mathrm{n}^{\circ}$ 5, p. 61-67.

BENNIS, W. (1982), «Leadership transforms vision into action», Industry Week, vol. 213 , p. 54-56.

BENNIS, W. et B. NANUS (1985), Diriger: les secrets des meilleurs leaders, Paris, InterÉditions.

BENNIS, W. (1990a), Why Leaders can't Lead, the Unconscious Conspiracy Continues, San Francisco, Jossey-Bass Publishers.

BENNIS, W. (1990b), «Managing the dream: leadership in the 21st Century », dans Training, vol. $27, \mathrm{n}^{\circ} 5$, p. 43-48.

BIALÈS, C. (1988), L'analyse statistique des données: outil statistique appliqué au marketing et à la gestion, Paris, Chotard et Associés Éditeurs.

BIRD, B. (1988), «Implementing entrepreneurial ideas : the case for intention », Academy of Management Review, vol. 13, $\mathrm{n}^{\circ} 3$, p. 442-453.

BIRD, B. et M. JELINEK (1988), «The operation of entrepreneurial intentions », Entrepreneurship Theory and Practice, vol. 13, $\mathrm{n}^{\circ} 2$, p. 21-29.

Blalock, H.M. (1979), Social Statistics, New York, McGraw-Hill.

CARRIÈRE, J.B. (1990), «La vision stratégique en contexte de PME: cadre théorique et étude empirique », Revue Internationale PME, vol. 3, nos 3-4, p. 301-325.

CARRIÈRE, J.B. (1989), «Strategic vision : an empirical study», Communication présentée à la $V^{e}$ Conférence annuelle du Conseil international de la petite entreprise, Windsor, Ontario, novembre.

CONGER, J.A. et R.N. KANUNGO (1988), «Behavioral dimensions of charismatic leadership », dans J.A. Conger, R.N. Kanungo et ass., Charismatic Leadership : The Elusive Factor in Organizational Effectiveness, San Francisco, Jossey-Bass Publishers, p. 78-97.

CONGER, J.A. (1990), «The dark side of leadership», Organizational Dynamics, vol. 19, $\mathrm{n}^{\circ} 2$, p. 44-55.

D’AMBoISE, G. et Y. GASSE (1989), «Vision stratégique et activités de planification: pour un modèle différent de recherche sur la PME, Communication présentée au XXXIVe Congrès du Conseil international de la petite entreprise, Québec, 21-23 juin.

D’ AMBoise, G. et J.M. Nkongolo B. (1993), «La vision stratégique des propriétairesdirigeants de PME: un essai d'identification et de mesure empirique », dans J.M. Toulouse (éd.), Entrepreneurship: Rapport du Congrès Annuel du groupe d'intérêt Entrepreneurship de l'ASAC, vol. 14, $\mathrm{n}^{\circ} 21$, Lac Louise, Alberta, 30 mai-2 juin, p. 54-78. 
D'Hainaut, L. (1978), Concepts et méthodes de la statistique, tome 2, Bruxelles, Éd. Labor; Paris, Fernand Nathan.

DESLAURIERS, J.P. (1991), Recherche qualitative: guide pratique, Montréal, McGrawHill.

DRUCKER, P. (1981), « Behind Japan's success », Harvard Business Review, vol. 59, $\mathrm{n}^{\circ} 1$, p. 83-90.

FILION, L.J. (1990), «Vision and relations : elements for an entrepreneurial metamodel », dans N.C. Churchill et al., Frontiers of Entrepreneurship Research: Proceedings of the Tenth Annual Babson College Entrepreneurship Research Conference, Babson Park, Massachussets, 24-29 avril, p. 57-71.

FORTUNE (1981), 15 juin.

GibB, A. et M. SCOTT (1985), «Strategic awareness, personal commitment and the process of planning in the small business », Journal of Management Studies, vol. $22, \mathrm{n}^{\circ} 6$, p. $599-631$.

GLUCK, F.W. (1984), «Vision and leadership», Interfaces, vol. 14, n 1, p. 10-18.

GrinYer, P.H., D.G. MAYES et P. MCKIERNAN (1988), Sharpbenders: The Secrets of Unleashing Corporate Potential, Oxford, Basil Blackwell Ltd.

HAAHTI, A.J. (1989), Entrepreneurs' Strategic Orientation: Modeling Strategic Behavior in Small Industrial Owner-managed Firms, Acta Academiæ Economicæ Helsingiensis, Series A : 64, The Helsinki School of Economics and Business Administration, Helsinki.

HAMBriCK, D.C. et L.A. CrozIER (1985), «Stumblers and stars in the management of rapid growth », Journal of Business Venturing, vol. 1, p. 31-45.

HrebiniAK, G.L., F.W. JOYCE et C.C. SNOW (1989), «Strategy, structure and performance: past and future research», dans C.C. Snow (éd.), 1989, Strategy, Organization, Design, and Human Ressource Management, London, JAI Press Inc, p. 3-54.

KEYS, J.B. et T.R. MiLler (1984), «The japanese management theory jungle», Academy of Management Review, vol. 9, $\mathrm{n}^{\circ} 2$, p. 342-353.

LES AFFAIRES (1991), Montréal, 19 octobre.

Livian , Y.F., V. RAMUS et R. PALIARD (1989), «Les recherches sur l'évaluation des projets de création d'entreprises, note de synthèse», Document n ${ }^{\circ} 6$, Groupe ESC, Lyon.

Mialaret, G. (1991), Statistiques appliquées aux sciences humaines, Paris, Presses universitaires de France.

MICT (1991), Le secteur manufacturier et le commerce au Québec en 1991, Direction de la conjoncture industrielle, Québec. 
MICT (1990), Les PME au Québec, état de la situation en 1989, Direction générale des politiques industrielles, Québec.

MiLLER, R. (1985), «La stratégie d'entreprise et la gestion des ressources humaines », Relations industrielles, vol. $40, \mathrm{n}^{\circ} 1, \mathrm{p} .68-86$.

Morgan, G. (1988), Riding the Waves of Change: Developing Managerial Competencies for a Turbulent World, San Francisco, Jossey-Bass Publishers.

MORRIS, E. (1987), «Vision and Strategy », The Journal of Business Strategy, vol. 8, $\mathrm{n}^{\circ} 2$, p. 51-58.

Moss KANTER, R. (1983), The Change Masters: Innovation for Productivity in the American Corporation, New York, Simon \& Schuster.

NADEAU, M.A. (1988), L'évaluation de programme: théorie et pratique, Québec, Les Presses de l'Université Laval.

Pascale, R.T. et A.G. Athos (1984), Le management est-il un art japonais? Paris, Les Éditions d'Organisation.

PETERS, T. (1988), Le chaos management: manuel pour une nouvelle prospérité de l'entreprise, Paris, InterÉditions.

PETERs, T. et R. WATERMAN (1983), Le prix de l'excellence, Paris, InterÉditions.

PIETTe, F. (1991), «Où va la PME?»PME, vol. 7, n 8, p. 8-10.

PrescotT, J.E., A.K. Kohli et N. Venkatraman (1986), «The market shareprofitability relationship : an empirical assessment of major assertions and contradictions », Strategic Management Journal, vol. 7, $\mathrm{n}^{\circ}$ 4, p. 377-394.

RICHARDS, D. et S. ENGEL (1986), «After the vision: suggestions to corporate visionaries and vision champions», dans J.D. Adams (éd.), Transforming Leadership : From Vision to Results, Virginia, Miles River Press, Alexandria, p. 199-214.

RobBins, S.R. et R.B. DUNCAN (1988), « The role of the CEO and top management in the creation and implementation of strategic vision », dans D. Hambrick (éd.), The Executive Effect: Concepts and Methods for Studying Top Managers, London, JAI Press Inc, p. 205-233.

RoBINSON, Jr R.B. (1983), «Measures of small firms effectiveness for strategic planning research», Journal of Small Business Management, vol. 21, $\mathrm{n}^{\circ} 2$, p. 22-29.

Robinson, Jr R.B., J.A. PeARCE II, G.S. Vozikis et T.S. MEscon (1984), «The relationship between stage of development and small firm planning and performance », Journal of Small Business Management, vol. 22, $\mathrm{n}^{\circ} 2$, p. 45-52.

ROCKEY, E.H. (1986), «Envisioning new businesses: how entrepreneurs perceive the benefits of vizualization? ?, dans R. Ronstadt, J. Hornaday, R. Peterson et K.H. Vesper (éd.), Frontiers of Entrepreneurship Research 1986, p. 344-360, Babson College, Wellesley, Mass. 
SAShKIn, M. (1988), «The visionary leader», dans J.A. Conger et al., Charismatic Leadership, San Francisco, Jossey-Bass Publishers, p. 122-160.

SAShKIN, M. (1986), «True vision in leadership », Training and Development Journal, vol. $40, \mathrm{n}^{\circ} 5$, p.58-61.

Siegel, S. et N.J. Castellan Jr (1988), Nonparametric Statistics for Behavioral Sciences, New York, McGraw-Hill.

SORGE, A. et M. MAURICE (1990), «The societal effect in strategies and competitiveness of machine-tool manufacturers in France and Germany », The International Journal of Human Resource Management, vol. 1, $\mathrm{n}^{\circ}$ 2, p. 141-172.

Thurston, P.H. (1983), «Should smaller companies make formal plans?» Harvard Business Review, septembre-octobre, p. 162-188.

Venkatraman, N. et V. RAMANUJAN (1986), «Measurement of business performance in strategy research: a comparison of approaches », Academy of Management Review, vol. $11, \mathrm{n}^{\circ} 4$, p. 801-814.

WILKINS, A.L. (1989), Developing Corporate Character: How to Successfully Change an Organization without Destroying it, San Francisco, Jossey-Bass Publishers.

Wils, T., C. LABELle, G. GuÉRIN et J.Y. LE LOUARN (1989), «La gestion stratégique des ressources humaines : un reniement du rôle social de l'entreprise ? Relations industrielles, vol. $44, \mathrm{n}^{\circ} 2$, p. 354-375.

YANG, C.Y. (1986), «Abus et faiblesses du management japonais », Harvard-L'Expansion, été, p. 82-89. 